\title{
A REDE DE POLÍTICAS DE SAÚDE PÚBLICA E A COOPERAÇÃO SUL-SUL: OS CASOS DE MOÇAMBIQUE E ANGOLA
}

Paulo Esteves

Professor do Instituto de Relações Internacionais da PUC-Rio e pesquisador do BRICS Policy Center, do CNPq e da Faperj. Rio de Janeiro, RJ, Brasil. Email < esteves_paulo@puc-rio.br >

Geovana Zoccal Gomes Mestre em Relações Internacionais (2013) pela PUC-Rio. Pesquisadora do BRICS Policy Center. Rio de Janeiro, RJ. Brasil.Email < geovana@bricspolicycenter.org >

\section{João Moura Fonseca}

Mestre em Relações Internacionais pela PUC-Rio. Consultor do Banco Mundial, em Moçambique e Pesquisador Associado do BRICS Policy Center. Dar es Salaam, Tanzânia.Email <joaomouraf@gmail.com>

http://dx.doi.org/10.1590/ 0102-6445199-230/98

O envolvimento brasileiro na cooperação internacional para o desenvolvimento teve início logo na própria constituição do campo no final da década de 1940, mas passa por constantes transformações desde então. No pós-guerra, foi receptor de um grande número de projetos da cooperação Norte-Sul, com foco na oferta de infraestrutura e de serviços públicos. Contudo, nos últimos anos passou a desempenhar papel-chave no estabelecimento de parcerias na cooperação Sul-Sul (CSS), ocupando a posição dual de país receptor e promotor de cooperação. A articulação com países do Sul global para um desenvolvimento mais autônomo ganhou força no Brasil primordialmente ao longo dos dois mandatos presidenciais de Luiz Inácio Lula da Silva, entre 2003 e 2010. Nesse período, a política externa brasileira conquistou ênfase autonomista, transformando a estratégia de inserção do país na política internacional.

De acordo com relatório oficial do governo acerca da cooperação brasileira para o desenvolvimento, entre os anos de 2005 e 2009, o investimento brasileiro na CSS passou de 384,2 milhões de reais para mais de 724 milhões de 
reais (Ipea/ABC, 2010). O portfólio da Agência Brasileira de Cooperação (ABC) em 2003 contava com apenas 23 novos projetos; já em 2009, esse número saltou para 413 (Cabral e Weinstock, 2010). Nesse contexto, o continente africano destaca-se com grande número de países parceiros e projetos destinados à região. Entre 2001 e 2010, o Programa Estudante-Convênio (PEC-G), que representa cerca de $80 \%$ dos recursos do Ministério da Educação destinados à cooperação internacional, ofereceu quase 5 mil bolsas a estudantes africanos (Brasil, 2013). Além da educação, os dois setores que se destacam na cooperação internacional com países africanos são agricultura e saúde pública.

Para a realização de tais projetos, a $\mathrm{ABC}$ - que tem como mandato negociar, coordenar, implementar e acompanhar os programas e projetos brasileiros de cooperação técnica - estabelece parcerias com agências executoras, como a Empresa Brasileira de Pesquisa Agropecuária (Embrapa), a 200 Fundação Oswaldo Cruz (Fiocruz) e o Serviço Nacional de Aprendizagem Industrial (Senai) - nos setores de agricultura, saúde e educação profissional, respectivamente. Assim, uma análise abrangente do envolvimento brasileiro na CSS só é possível quando levado em consideração o papel que tais agências adquirem nesse campo.

$\mathrm{O}$ argumento desenvolvido pelos autores ao longo de pesquisas de campo realizadas em Moçambique, Angola e Brasil $^{1}$, aqui articulado, baseia-se em uma perspectiva de bottom-up, que leva em conta trajetória e ações da ABC, mas enfatiza aquelas das agências executoras da cooperação.

\footnotetext{
1 Como parte das atividades conduzidas pelo Brazilian-SSC Project, projeto de pesquisa realizado pelo BRICS Policy Center com o apoio do UK Department for International Development. Os autores realizaram três visitas a Moçambique (entre 2013 e 2015) e duas a Angola (entre 2014 e 2015), além de grande número de entrevistas no Brasil. Ao todo, mais de 70 profissionais representantes de agências doadoras multilaterais e bilaterais, academia, sociedade civil e governos nacionais foram entrevistados. Adicionalmente, foram realizadas três oficinas de validação dos resultados nas cidades do Rio de Janeiro, Maputo e Luanda.
} 
Assim, o fundamento para a análise torna-se o que chamamos de redes setoriais de políticas públicas. Este artigo parte da constituição do que chamamos de Rede de Políticas de Saúde Pública para a análise das ações de cooperação brasileira no setor respectivo em Moçambique e Angola.

Moçambique, um dos principais parceiros da cooperação brasileira, contava, em 2013, segundo a ABC (O Brasil..., 2013), com catorze projetos em andamento. $\mathrm{Na}$ área da saúde, a parceria com Moçambique conta com um dos projetos mais conhecidos da cooperação brasileira - a Sociedade Moçambicana de Medicamentos (SMM). O país é um dos pontos focais das ações brasileiras de cooperação na área da saúde pública.

A cooperação brasileira com Angola também é significativa. No âmbito da cooperação para o desenvolvimento, Angola ocupava, em 2012, a quinta posição em termos de volume da cooperação técnica recebida do Brasil (O Brasil..., 2013). Tanto Moçambique como Angola são parceiros do Brasil na Rede de Bancos de Leite Humano. Em Angola, o Proforsa (Projeto de Fortalecimento do Sistema de Saúde em Angola) é um dos projetos mais conhecidos nesse setor. A partir de uma iniciativa trilateral com o Japão, o Proforsa teve como objetivo a capacitação individual e institucional para a saúde pública angolana, como se verá a seguir.

Como já dito, para tratar da CSS brasileira em saúde, este artigo tem como ponto de partida uma análise de Rede de Políticas de Saúde Pública no Brasil. Conhecer os processos de constituição e consolidação da Rede embasa o argumento de que o engajamento dos seus agentes em projetos de CSS deve ser compreendido por um viés mais amplo de extensão internacional da própria Rede. Essa extensão contribui para o posicionamento internacional do governo brasileiro no campo da saúde global, uma vez que carrega consigo escolhas constitucionais e de políticas que distinguem a Rede como tal. O segundo movimento do texto busca 
explorar os portfólios de projetos de CSS em saúde em Angola e Moçambique e relacioná-los à extensão internacional da Rede de Políticas de Saúde Pública através do Plano Estratégico de Cooperação em Saúde da Comunidade dos Países de Língua Portuguesa (CPLP). Tal movimento nos permite propor uma série de conclusões que indicam, do ponto de vista da formulação de políticas, a necessidade de consolidação do que os cooperantes brasileiros do campo da saúde denominam cooperação estruturante. Ademais, nas conclusões, apontamos, ainda, a necessidade de trabalhos de campo sobre a extensão internacional da Rede, de forma a permitir a compreensão das formas de articulação entre cooperantes brasileiros e seus parceiros na África lusófona.

\section{A trajetória da cooperação Sul-Sul brasileira e suas redes de políticas públicas}

É comum o tratamento da cooperação brasileira em ter-

202 mos de transferência de políticas públicas que lograram maior ou menor sucesso no Brasil ${ }^{2}$. Como buscaremos demonstrar, contudo, mais do que transferência de políticas, podemos caracterizar a cooperação brasileira - pelo menos no que concerne ao caso da saúde pública - como uma extensão internacional da Rede Brasileira de Políticas de Saúde Pública. De fato, nos dois lados do Atlântico, informantes relacionaram a cooperação em saúde à extensão internacional de suas respectivas redes. Como cooperantes, os agentes brasileiros caracterizam, com frequência, seu trabalho como uma extensão internacional

\footnotetext{
2 A despeito dos ganhos analíticos que oferece o tratamento da CSS em termos de transferências de políticas, tal abordagem apresenta, muitas vezes, limites no que se refere, de um lado, à relação entre a transferência de políticas públicas e o ordenamento do sistema internacional e, de outro lado, aos condicionantes sociais e políticos do processo de transferência. Sobre a aplicação dessa abordagem ao caso brasileiro, ver Hochman (1988), Lana e Evans (2004), Melo (2004), Hochman (2007), Sugyiama (2011), Faria (2012) e Milani e Lopes (2014).
} 
de escolhas constitucionais ${ }^{3}$, programas e projetos que conduzem em casa.

A referida extensão internacional diz respeito não apenas à transferência de políticas específicas, mas à estruturação de sistemas setoriais e à promoção, no âmbito internacional, de escolhas constitucionais produzidas no ambiente doméstico. A extensão internacional das redes de políticas domésticas ${ }^{4}$ pode fortalecer ou contribuir para a produção de escolhas constitucionais e legitimar políticas e projetos executados no Brasil ${ }^{5}$.

Nesse sentido, esforços de cooperação internacional das redes brasileiras de políticas públicas ultrapassam a dimensão da transferência de políticas consideradas exitosas. De fato, a extensão internacional das redes nacionais reveste-se de importância estratégica para a consolidação de sistemas setoriais e políticas domésticas fundamentados em escolhas constitucionais em operação no Brasil. As extensões internacionais são, assim, partes constitutivas da dinâmica de redes de políticas públicas, combinando: (i) a atuação em arenas políticas multila-

3 Embora referenciado na obra de Buchanan, o uso que aqui se faz do conceito de "escolha constitucional" é ligeiramente diferenciado. Segundo Buchanan, escolhas constitucionais dizem respeito ao estabelecimento de regras acerca da produção de regras, sejam elas as regras de competição política, diversos constrangimentos regulatórios ou arranjos institucionais que constituem a ordem social (Buchanan e Tullock, 1962; Brennan e Buchanan, 1985; Buchanan, 1987, 1990). Para além da definição das regras de competição política e proteção de propriedade, pode-se considerar que o estabelecimento do caráter público de determinados bens e a definição de direitos constituem um conjunto de escolhas constitucionais que constrangerão o comportamento e as decisões dos agentes em momento pós-constitucional. Ora, escolhas constitucionais implicam o estabelecimento de direitos de propriedade, bens públicos e direitos individuais, e resultam na configuração de sistemas e políticas setoriais no plano doméstico. Dessa forma, escolhas são objeto de disputas continuadas, tanto nacional como internacionalmente.

4 A relevância das redes de políticas domésticas para a CSS do Brasil já é apontada em Leite, Suyama e Waisbich (2013).

${ }^{5}$ Os casos de saúde e segurança alimentar são elucidativos. A Rede de Políticas de Saúde Pública no Brasil conseguiu garantir a constitucionalização do direito à saúde e buscou, particularmente na questão do acesso a fármacos, a arena multilateral para garantir a eficácia de políticas públicas específicas. A rede de segurança alimentar, por seu turno, foi capaz de influenciar decisivamente a formulação e implementação das políticas desse setor. Contudo, buscou a arena multilateral para constitucionalizar o direito à saúde como um direito universal e, portanto, da cidadania brasileira. 
terais; (ii) a constituição e / ou participação em arenas minilaterais ou regionais; e (iii) a cooperação para o desenvolvimento ${ }^{6}$.

A literatura de análise de redes de políticas (policy network analysis) representa um ponto de entrada possível para uma investigação mais aprofundada do setor da saúde no Brasil e de suas articulações internacionais ${ }^{7}$. Tal literatura nos permite compreender as dinâmicas internas a cada um dos setores pesquisados, bem como os processos de decisão e implementação de escolhas constitucionais e políticas públicas. Esperamos demonstrar, ainda, que esses processos podem ser percebidos não apenas no âmbito doméstico, mas também em extensões internacionais, que podem assumir a forma de cooperação para o desenvolvimento.

Redes de políticas são arranjos estáveis que reúnem uma variedade de atores, cujas relações rotineiras são marcadas por interdependência, pela partilha de interesses comuns e recursos necessários para a promoção de políticas 204 específicas (Börzel, 1998). O caso do setor da saúde pública é exemplo dos processos de constituição, dinâmica e extensão internacional de redes de políticas no Brasil.

\section{A cooperação brasileira em saúde pública}

A constituição da Rede de Políticas de Saúde Pública relaciona-se à dinâmica do movimento sanitarista ao longo do

\footnotetext{
6 Enquanto arenas e normas multilaterais supõem princípios generalizados de conduta e reciprocidade difusa, arranjos e arenas minilaterais são válidos apenas para seus participantes. Como clubes, nos arranjos minilaterais "a participação é restrita, com os membros privilegiados para agir como definidores de agenda na formulação de políticas; além disso, proporciona bens exclusivos a seus membros" (Naim, 2009, Cooper e Farooq, 2015).

7 Analiticamente, conforme a definição de Marsh e Rhodes (1992), os policy networks são conceitos adequados para tratar de níveis intermediários, uma vez que nos permitem relacionar os níveis micro vinculados à interesses particulares e os níveis macro relativos a decisões de governos acerca de políticas. De acordo com Rhodes (2006), as redes de políticas possuem níveis distintos de integração que nos propiciam identificar redes altamente integradas que funcionam como comunidades a redes temáticas, nas quais o grau de consenso é bastante escasso.
} 
regime militar no Brasil ${ }^{8}$. Sua origem vincula-se à construção, ainda na década de 1950, dos departamentos de medicina preventiva e à incorporação em 1968 desse campo de conhecimentos aos currículos das faculdades de medicina no país (Escorel, 1999; Escorel, Nascimento e Edler, 2005). Durante os anos 1970 e início dos 1980, os departamentos de medicina preventiva das universidades brasileiras tornaram-se palco de disputas entre perspectivas concorrentes acerca do objeto da saúde e das políticas necessárias para a sua promoção. Foi nesse período que se estabeleceram algumas das perspectivas e dos conceitos-chave em torno dos quais se organizaria a Rede: o conceito de determinantes sociais da saúde (Buss e Pellegrini Filho, 2007) e a perspectiva que associa a promoção da saúde a mecanismos democráticos de participação e controle social. O desenvolvimento dessa perspectiva sob o regime autoritário deu-se nos poucos nichos institucionais em que os profissionais da saúde vinculados ao movimento sanitário poderiam atuar ${ }^{9}$. Ainda na segunda metade da década de 1970, foram criadas duas outras organizações que contribuíram para o avanço dos debates em torno da reforma sanitária e da democratização da saúde: o Centro Brasileiro de Estudos da Saúde (Cebes) e a Associação Brasileira de Pós-Graduação em Saúde Coletiva (Abrasco) ${ }^{10}$.

\footnotetext{
8 Apesar da ênfase que se dá à dinâmica do movimento sanitarista na década de 1970, as origens do movimento datam do início do século XX, quando a saúde era tratada tanto como um problema quanto como um caminho civilizador. $\mathrm{Na}$ primeira metade do século XX, o movimento ganha especial relevância à luz das iniciativas de construção do Estado e de integração do território nacional no Brasil (Lima, Fonseca e Hochman, 2005).

9 Segundo Sarah Escorel, três instituições teriam contribuído para a estruturação do movimento sanitarista em meados dos anos 1970: o Centro Nacional de Recursos Humanos do Instituto de Pesquisas Econômicas e Aplicadas (CNRH-Ipea), a Financiadora de Estudos e Projetos (Finep) e o Programa de Preparação Estratégica de Pessoal de Saúde da Organização Pan-Americana da Saúde (Ppreps-Opas) (Escorel, Nascimento e Edler, 2005).

${ }^{10}$ O Cebes surgiu em 1976 a partir do curso de Saúde Pública da Universidade de São Paulo (USP). O Centro foi responsável pela criação da revista Saúde em Debate, que viria a se tornar importante espaço de articulação de propostas para a reforma sanitária. A Abrasco, fundada em 1979, reuniu os programas de pós-graduação em
} 
Ao final da década de 1970, já era possível divisar os contornos do que viria a ser a Rede. Com efeito, naquele momento, diversos agentes, incluindo médicos, trabalhadores do sistema de saúde, agentes públicos e organizações civis encontraram arenas específicas de interação como o Cedes e a Abrasco, articulando-se em torno de uma concepção de saúde pública que enfatizava os seus determinantes sociais, bem como a necessidade de democratização do sistema de saúde, elemento que estaria vinculado diretamente à maior participação social em processos decisórios naquele setor. No início dos anos 1980, ao longo do processo de democratização no Brasil, esses agentes lograram ampliar seus espaços de participação junto ao Estado, tomando parte em simpósios e conferências e construindo uma agenda própria para a reforma do sistema de saúde pública ${ }^{11}$. Já se pode identificar nesse período uma Rede de Políticas de Saúde Pública, caracterizada por significativa integração. Tal integração manifestava-se na partilha de 206 valores comuns, o que limitava seu número de participantes e potencializava o volume e qualidade de suas interações. A análise de Sarah Escorel nos permite compreender, ainda, que, na primeira metade da década de 1980, os participantes da Rede construíram uma agenda comum de reformas para o setor, que, ao fim e ao cabo, era expressão de um conjunto de preferências políticas compartilhadas. Assim, segundo a autora,

[...] a década de 1980 se inici[ou] com um movimento cada vez mais forte de contestação ao sistema de saúde governamental. As propostas alternativas ao modelo oficial de atenção à saúde caracterizavam-se pelo apelo à democratização do sistema, com participação popular, a universalização dos

\footnotetext{
saúde coletiva e saúde pública do Brasil. Com o apoio dessa associação foram fortalecidos os programas de residência em medicina preventiva e social, que à época se expandiam pelo país (Escorel, Nascimento e Edler, 2005).

${ }^{11}$ Exemplos: I Simpósio sobre Política Nacional de Saúde, em 1979, organizado pela Câmara dos Deputados; e 7ª Conferência Nacional de Saúde, em 1980.
} 
serviços, a defesa do caráter público do sistema de saúde e a descentralização (Escorel, Nascimento e Edler 2005, p. 71).

Com cerca 4 mil participantes e mil delegados, a $8^{\text {a Con- }}$ ferência Nacional de Saúde, em 1986, assinala o momento no qual se inicia o processo de debate constitucional sobre saúde pública no Brasil. A convocação da conferência já se deu a partir das perspectivas partilhadas pela Rede. Seus resultados apontam para o relativo consenso já existente em torno das perspectivas promovidas pelos participantes da Rede no interior da arena de saúde pública no país. De fato, a conferência aprovou o texto final, que consagra uma perspectiva abrangente de saúde, enfatizando seus determinantes sociais.

Em seu sentido mais abrangente, a saúde é a resultante das condições de alimentação, habitação, educação, renda, meio ambiente, transporte, emprego, lazer, liberdade, acesso e posse da terra e acessos a serviços de saúde. É assim, antes de tudo, o resultado das formas de organização social da produção, as quais podem gerar grandes desigualdades nos níveis de vida (Brasil, 1986, p. 4).

Na mesma perspectiva, o texto final da conferência defende que a saúde deveria se constituir em direito da cidadania, cuja garantia e provisão seriam obrigações do Estado:

[...] direito à saúde significa a garantia, pelo Estado, de condições dignas de vida e acesso universal e igualitário às ações de promoção, proteção e recuperação da saúde, em todos os seus níveis, a todos os habitantes do território nacional, levando ao desenvolvimento pleno do ser humano em sua individualidade (Brasil, 1986, p. 4).

A constitucionalização do direito à saúde e a construção do aparato institucional para sua garantia são em muito decor- 
rentes das ações da $\operatorname{Rede}^{12}$. A constitucionalização dos valores compartilhados entre os participantes da Rede e sua capacidade de negociar a adoção de parte significativa de suas preferências como políticas públicas, bem como os mecanismos necessários para sua implementação, são indicativos do enraizamento social da Rede. Ademais, a Constituição Federal de 1988 e a garantia do direito universal à saúde são, portanto, indicativos da influência da Rede sobre a própria arena política da saúde, em vista das dificuldades que se apresentariam para efetiva promoção dos princípios constitucionalizados.

A série de entrevistas realizadas ao longo da pesquisa permitiu identificar a percepção, compartilhada por muitos agentes do setor, de que, no Brasil, as esferas da política doméstica, política internacional no setor de saúde e cooperação para o desenvolvimento possuem vínculos inextricáveis. Com efeito, a narrativa prevalente entre agentes da comunidade de saúde pública no Brasil relaciona o pro208 cesso de escolha constitucional da saúde como um direito à atuação internacional de agentes oficiais brasileiros no campo da saúde. A construção de um sistema de saúde pública orientado pelos princípios da universalidade, integralidade, equidade, descentralização e controle social não é tratada, apenas, como resultado de disputas político-sanitárias domésticas. De acordo com essa narrativa, tal escolha consolidou-se através de um processo de resistência contra as reformas orientadas ao mercado, impostas pelas instituições financeiras internacionais, particularmente pelo Banco Mundial ${ }^{13}$, e de promoção de normas internacionais relacionadas ao direito à saúde e à construção/provisão de bens públicos globais relacionados.

12 Com a Constituição de 1988 e com a regulamentação do Sistema Único de Saúde (SUS) através de lei complementar, asseguravam-se os princípios advogados pelo Movimento Sanitarista e pela Rede: universalidade, integralidade, equidade, descentralização e controle social.

${ }^{13}$ Ver World Bank (1987), Mattos, (2001), Escorel (1999), Escorel, Nascimento e Edler (2005) e Almeida (2006). 
De fato, é nesse contexto que tem lugar a extensão internacional da Rede e o processo de institucionalização de seu relacionamento com a Comunidade de Política Externa Brasileira $^{14}$. Essa extensão internacional se desdobra em três dimensões: multilateral, minilateral e cooperação Sul-Sul. Na primeira dimensão, a atuação em fóruns internacionais e junto a organizações intergovernamentais, incluindo a Organização Mundial da Saúde (OMS), Organização Mundial do Comércio (OMC) e a Assembleia Geral das Nações Unidas, foi marcada pela promoção de princípios como acesso universal a cuidados de saúde de qualidade e medicamentos. A segunda dimensão manifesta-se na defesa dessas posições em arenas políticas como a Organização Pan-Americana de Saúde (OPAS), a União das Nações Sul-Americanas (Unasul) e a Comunidade dos Países de Língua Portuguesa (CPLP) (Buss, 2011). Nessas arenas, para além da promoção dos princípios anteriormente referidos, a dinâmica da Rede tem lugar na construção de planos de ação comuns e de redes estruturantes, com vistas ao fortalecimento dos sistemas nacionais de saúde.

No caso específico da CPLP (2009), ministros da saúde lograram construir um plano estratégico para o setor, objetivando fortalecer sistemas nacionais e assegurar acesso universal a serviços de saúde de qualidade. O plano compreendia sete eixos estratégicos: Força de Trabalho, Informação e Comunicação, Investigação em Saúde, Complexo Produtivo da Saúde, Vigilância Epidemiológica e Monitoramento da Situação de Saúde, Emergências e Desastres Naturais e Promoção e Proteção da Saúde; e quatro redes estruturantes: Institutos Nacionais de Saúde, Escolas Nacionais de Saúde Pública, Escolas Técnicas

\footnotetext{
${ }^{14}$ A literatura sobre redes de políticas oferece um quadro analítico que permite discriminar tipos de redes e seus diferentes graus de integração e consistência interna. Rhodes identifica cinco variáveis que determinariam o nível de integração de uma rede de políticas: (i) número de participantes; (ii) frequência de interações entre os participantes; (iii) consistência em relação a valores, critérios de pertencimento e resultados esperados; (iv) consenso em relação a preferências de políticas; e, (v) interdependência entre os participantes (Rhodes, 1988, 2006; Marsh e Rhodes, 1992).
} 
de Saúde e Bancos de Leite Humano. Adicionalmente, o Ministério da Saúde (MS) e a Fiocruz expandiram para os países africanos de língua portuguesa a Rede de Bancos de Leite Humano. A construção de redes de cooperação Sul-Sul e a execução de projetos de cooperação para o desenvolvimento no campo em questão podem ser compreendidas, assim, à luz da construção mais ampla de coalizões no campo da saúde global e do fortalecimento de sistemas domésticos de saúde pública.

A extensão internacional da Rede tem suas ações organizadas em torno de três noções vinculadas entre si: diplomacia da saúde, complexo industrial da saúde e cooperação estruturante. Com efeito, agentes brasileiros frequentemente lançam mão do conceito de diplomacia da saúde ao discutir respostas a problemas transnacionais de saúde emergentes (Kickbuch, Silberschmidt e Buss, 2007; Buss e Ferreira, 2010). A noção de diplomacia da saúde proporcionou um guarda-chuva conceitual para melhor articulação entre o 210 Ministério das Relações Exteriores (MRE) e o Ministério da Saúde (MS). Facilitou também o maior engajamento internacional de instituições tradicionalmente envolvidas apenas em questões de saúde em âmbito nacional. A noção é, ainda, frequentemente apresentada como uma alternativa à suposta separação entre a política externa e as metas de ajuda ao desenvolvimento em geral, associadas à ajuda do Norte (Russo, Cabral, e Ferrinho, 2013). Vale notar, contudo, que, na percepção de alguns entrevistados, a noção de diplomacia da saúde e as ações que nela se justificam têm criado algumas tensões entre a Rede e a comunidade de política externa, frequentemente associadas a disputas burocráticas relacionadas à responsabilidade última sobre a política externa do Brasil ${ }^{15}$.

\footnotetext{
${ }_{15}$ A frequente interação entre o MRE e o MS levou a institucionalizar a Assessoria Internacional (Aisa/MS). Contudo, a despeito da já consolidada Aisa, vários informantes insistiram na centralidade do relacionamento entre as lideranças de cada um dos ministérios. A dependência de relações ad hoc que não necessariamente se dão através dos mecanismos de coordenação interministerial indica um processo inconcluso de ajuste entre a rede e a comunidade de política externa.
} 
Ao defender as escolhas constitucionais de 1988 não apenas pelas lentes dos direitos, agentes da saúde afirmam que ganhos de produtividade resultam de um sistema de saúde eficaz e abrangente. Em outras palavras, uma população saudável traduz-se em uma força de trabalho mais produtiva (Fiocruz, 2012). O desenvolvimento de um complexo industrial da saúde - baseado em uma estratégia nacional de desenvolvimento que enfatiza a redução da dependência econômico-produtiva em setores estratégicos, como a biotecnologia e equipamentos eletrônicos - é apresentado como pré-requisito ao bom funcionamento do sistema de saúde, gerando concomitantemente efeitos econômicos positivos. A dinâmica é descrita por Gadelha (2006), entre outros autores, como um ciclo virtuoso construído em torno do processo de retroalimentação entre a economia do país e sua saúde pública. De acordo com esse argumento, amplamente adotado pelo próprio governo brasileiro, o complexo industrial da saúde minimizaria riscos em relação ao alcance de objetivos da saúde pública do Brasil, em particular a universalidade, equidade e integralidade.

Essas aspirações manifestam-se na extensão internacional da Rede através do que seus participantes denominam cooperação estruturante. Ainda que já utilizada em outros setores da cooperação brasileira, a noção de cooperação estruturante é mais consolidada no âmbito da saúde, possuindo nuances relevantes. Essa teria como centro

[...] o fortalecimento institucional dos sistemas de saúde dos países parceiros, combinando intervenções concretas com a construção de capacidades locais e a geração de conhecimento, e ainda promovendo o diálogo entre atores, de forma a possibilitar que eles assumam o protagonismo na liderança dos processos no setor saúde e promovam a formulação autônoma de uma agenda para o desenvolvimento futuro na saúde (Almeida et al., 2010, p. 28). 
A cooperação estruturante brasileira em saúde é frequentemente contrastada com abordagens tradicionais "pautadas por programas verticais, vinculados a doenças específicas e que, aparentemente, teriam pouco impacto nos sistemas de saúde e nos resultados para as populações" (Almeida et al., 2010, p. 28). O tema será aprofundado nas seções a seguir dedicadas aos casos de Moçambique e Angola.

\section{A atuação da Rede de Políticas de Saúde Pública e a CSS em Moçambique e Angola}

\section{A cooperação em saúde com Moçambique ${ }^{16}$ \\ O sistema de saúde moçambicano tem apresentado} diversas melhoras nos últimos anos. A taxa de mortalidade infantil, taxa de mortalidade dos menores de 5 anos e a taxa de mortalidade materna têm diminuído de forma significa-

212 tiva. Contudo, desigualdades entre as regiões do país ainda são elevadas e o estado geral da saúde em Moçambique permanece abaixo dos padrões internacionais e da média africana. A prevalência do vírus HIV/Aids não é somente alta, mas crescente (Visser-Valfrey e Umarji, 2010).

O apoio de doadores do Norte e organizações internacionais ao setor da saúde moçambicano é coordenado pelo Grupo de Parceiros da Saúde (Health Partner's Group - HPG), cujos membros se encontram mensalmente. A OMS e a Unicef (Fundo das Nações Unidas para a Infância) são os mais influentes, apesar de instituições financiadoras globais, como a Aliança Mundial para as Vacinas e a Imunização (GAVI), desempenharem papel proeminente. Os Estados Unidos, através da Agência dos Estados Unidos para o Desenvolvi-

\footnotetext{
16 Os resultados apresentados sinteticamente nesta seção encontram-se no relatório Brazilian health and agricultural cooperation in Mozambique: an overview, publicado em: <http://bricspolicycenter.org/homolog/uploads/trabalhos/6549/ doc/970646912.pdf $>$.
} 
mento Internacional (Usaid) e do Centro para Controle e Prevenção de Doenças (CDC), são os maiores dentre os doadores no setor da saúde em Moçambique. Além disso, organizações não governamentais, como a Fundação Clinton e o Naima+ (Rede das ONGs internacionais que trabalham na área da Saúde e HIV em Moçambique), ocasionalmente participam das reuniões do grupo de coordenação. As ações do grupo devem, em princípio, estar alinhadas com os planos estratégicos para o desenvolvimento da saúde formulados pelo governo de Moçambique ${ }^{17}$. Apesar de profissionais brasileiros geralmente não participarem das reuniões do $\mathrm{HPG}^{18}$, as ações do grupo terminam por incidir sobre a cooperação brasileira para o desenvolvimento.

Informantes frequentemente apontaram para a centralidade do Plano Estratégico (2009-2012) de Cooperação em Saúde da CPLP (PECS/CPLP). O plano estratégico tinha como principal objetivo o fortalecimento de sistemas nacionais de saúde, de modo a garantir acesso universal a serviços de saúde de qualidade ${ }^{19}$. Além da rede estruturante estabelecida dentro do plano de saúde da CPLP, o MS e a Fiocruz expandiram para países africanos a Rede de Bancos de Leite Humano. De acordo com as entrevistas rea-

\footnotetext{
${ }^{17}$ Quais sejam: (i) Primeiro Plano Estratégico do Setor da Saúde - PESS I, desenvolvido em 2000 e implementado em 2002; (ii) PESS II, referente ao período de 2007 a 2012 e estendido por mais um ano; (iii) PESS III, que cobriu o período de 2014 a 2019. Além desses, vale mencionar também o Plano de Ação para a Redução da Pobreza, o Plano Nacional Integrado para o alcance dos ODMs 4 e 5, o Plano Estratégico para o Controle da Tuberculose, o Plano Estratégico Nacional de Resposta ao HIV e SIDA, o Plano de Desenvolvimento de Recursos Humanos da Saúde 2008-2015 e o Plano Estratégico do Sistema de Informação para a Saúde. ${ }^{18}$ Alguns informantes apontaram para os atritos do Brasil com o DAC, como a explicação para a falta de participação brasileira em tal estrutura de coordenação, mesmo que conte com a presença esporádica da representação brasileira no país, como no caso do grupo do setor agrícola em Moçambique. Outros informantes declararam pessoal desgosto com a atitude de doadores tradicionais durante tais encontros. Nenhuma explicação é completa, assim a ausência de recursos humanos adequados, tempo, e outros aspectos também devem ser considerados, pois contribuem para a frequente falta de participação de profissionais brasileiros em tais estruturas.

${ }^{19}$ Para mais informações, acessar: <http://www.cplp.org/id-1787.aspx>.
} 
lizadas em Moçambique, o PECS, cujos eixos estratégicos e redes estruturantes são apresentados no Quadro 1, constituiria o principal enquadramento para a cooperação entre esses países. Entrevistas indicaram também o papel capital do Brasil na definição de tais planos. O discurso do então Ministro José Gomes Temporão permite perceber como a construção do Plano estende para o ambiente minilateral (a CPLP) as escolhas constitucionais e de políticas tomadas no âmbito da Rede de Políticas de Saúde Pública brasileira.

O Brasil tem a perspectiva de contribuir muito firmemente para a consecução do PECS/CPLP. Quando o examinamos, percebemos a abrangência de seus propósitos, que vai da construção de redes de "instituições estruturantes" dos sistemas de saúde, como são: nossos próprios Ministérios e as redes de Escolas Técnicas de Saúde, de Escolas de Saúde 214 Pública, de Institutos Nacionais e de Centros de Instalação e Manutenção de Equipamentos, a um ambicioso programa de formação de recursos humanos, que inclui a rede de observatórios em recursos humanos em saúde; da vigilância epidemiológica e monitorização da situação de saúde vis-àvis os Objetivos de Desenvolvimento do Milênio ao reforço dos nossos sistemas de saúde - incluindo as estratégias da atenção primária integral de saúde e o acesso universal aos medicamentos essenciais - à cooperação em situações de emergências e desastres naturais e a priorização da promoção e proteção à saúde. Ademais, demonstrando grande senso de oportunidade, o Plano se debruça sobre o complexo produtivo da saúde para produzir vacinas, medicamentos, kits para diagnóstico e outros insumos para a saúde que, em tempos de crise econômica, pode ser um forte dinamizador da economia dos nossos países (Temporão, 2009). 


\section{Quadro 1}

Plano Estratégico de Cooperação em Saúde da CPLP:

Eixos Estratégicos e Redes Estruturantes

\begin{tabular}{|c|c|}
\hline \multirow{7}{*}{ 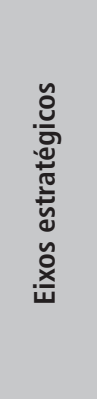 } & EE1 - Treinamento e desenvolvimento de mão de obra da área da saúde \\
\hline & EE2 - Informação e comunicação na saúde \\
\hline & EE3 - Pesquisa em saúde \\
\hline & EE4 - Desenvolvimento do complexo industrial da saúde \\
\hline & EE5 - Vigilância epidemiológica e monitoramento de situações da saúde \\
\hline & EE6 - Emergências e desastres naturais \\
\hline & EE7 - Promoção e proteção da saúde \\
\hline \multirow{4}{*}{ 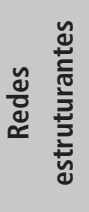 } & RE1 - Institutos nacionais da saúde \\
\hline & RE2 - Escolas nacionais de saúde pública \\
\hline & RE3 - Escolas técnicas de saúde \\
\hline & RE4 - Centros técnicos de instalação e manutenção de equipamentos \\
\hline
\end{tabular}

Fonte: CPLP (2009).

A principal contraparte da cooperação brasileira para o Ministério da Saúde de Moçambique (Misau) é a Fiocruz, descrita por um dos informantes do Misau como um ministério em si mesmo. A Fiocruz e o Instituto Nacional de Saúde de Moçambique (INS) definiram, em outubro de 2012, um plano de cooperação para os cinco anos seguintes, abrangendo as áreas de pesquisa, ensino, vigilância da saúde, informação, comunicação, histórico da saúde e sistemas de saúde, assim como atividades no âmbito da Rede Nacional dos Laboratórios de Saúde Pública e gestão do INS.

A Fiocruz está envolvida no apoio ao INS para a preservação da sua história institucional e arquivos. Esse projeto envolve uma revisão da história da saúde pública de Moçambique pós-independência e o desenvolvimento de uma revista eletrônica pronta para ser indexada internacionalmente. Além disso, a Fiocruz está apoiando a criação de 
um banco de leite no Hospital Central de Maputo. Considerado por muitos informantes o projeto mais bem-sucedido promovido pelo Brasil na cooperação internacional para o desenvolvimento, os bancos de leite são desenhados considerando a extensiva experiência acumulada pelo Brasil, que teve seu primeiro banco de leite implementado em 1943 (Anvisa, 2008).

Ademais, o Instituto Oswaldo Cruz, a Fiocruz e o INS de Moçambique estabeleceram parceria para criar um programa de mestrado em Ciências da Saúde para Moçambique. Os estudantes têm um orientador brasileiro e outro moçambicano, e passam três meses estudando no Brasil. Outro mestrado, em Sistemas de Saúde, foi desenhado em parceria com o INS, o Centro Internacional de Pesquisa para o Desenvolvimento (IDRC), do Canadá, a Escola Nacional de Saúde Pública (ENSP/Fiocruz) e o Centro de Pesquisas Aggeu Magalhães. Membros da Universidade Eduardo Mond216 lane foram incorporados ao corpo docente do programa.

O projeto mais conhecido da Fiocruz em Moçambique é a Sociedade Moçambicana de Medicamentos. Anunciada em 2003 pelo então Presidente Luiz Inácio Lula da Silva, o projeto está sob implementação há mais de uma década. A iniciativa relaciona-se à posição brasileira, tanto doméstica quanto externa, relativa ao direito de produção de medicamentos genéricos em contextos de epidemia. Moçambique tem contribuído com um espaço em Matola, cidade adjacente a Maputo, onde havia uma fábrica de produção de soros. A Agência Nacional de Vigilância Sanitária (Anvisa) e o Ministério da Saúde (MS) brasileiros fizeram uma parceria para apoiar a criação da Autoridade Regulatória de Medicamentos de Moçambique, que controlaria a qualidade e preço dos medicamentos, de acordo com diretrizes da OMS. Contudo, informantes disseram que o projeto foi arquivado após perder apoio político com mudanças do Misau. 
Entrevistas indicaram a existência de um projeto de caracterização da saúde oral e dental de Maputo pelo MS do Brasil, com o objetivo de criar condições para melhor planejamento e avaliação das ações e serviços públicos estomatológicos. Informantes apontam também para a parceria do MS com o Instituto Nacional do Câncer (Inca), com o objetivo de desenvolver o sistema de informações de Moçambique sobre a enfermidade e apoiar programas de prevenção ao câncer de colo do útero e de mama. Além disso, Bruyn (2014) menciona um projeto de cuidado comunitário em parceria com a Universidade de Brasília (UnB), para o qual o governo brasileiro teria comprometido 425 mil dólares, projeto que a equipe de pesquisa auferiu como concluído. Um informante mencionou uma atividade em parceria com a Universidade Federal do Ceará (UFC) e a Universidade Federal do Rio de Janeiro (UFRJ), incluindo um programa de intercâmbio com a escola de enfermagem da UFRJ. O mesmo informante apontou para a importância das bolsas de estudo brasileiras concedidas a estudantes moçambicanos na área da saúde, usualmente coordenadas pelo Ministério da Ciência e Tecnologia de Moçambique e emitidas pelo Conselho Nacional de Desenvolvimento Científico e Tecnológico (CNPq) e pela Coordenação de Aperfeiçoamento de Pessoal de Nível Superior (Capes), com a participação da Divisão de Temas Educacionais do Ministério das Relações Exteriores.

A breve descrição do portfólio de projetos de cooperação na área de saúde em Moçambique permite identificar seu enquadramento nos marcos estabelecidos no PECS/ CPLP. O Quadro 2 contrasta o portfólio de projetos em Moçambique aos eixos estratégicos PECS/CPLP, propiciando verificar o alto grau de consistência entre eles, o que nos leva a três conclusões preliminares:

1. A elaboração do PECS encontra-se intimamente vinculada às experiências da cooperação brasileira em saúde 
em países lusófonos. Muitos projetos são anteriores à essa elaboração, o que nos faz divisar uma dinâmica circular entre as experiências de campo da CSS brasileira e a formulação de estratégias mini e multilaterais.

2. A consistência entre, de um lado, as escolhas constitucionais e por políticas tomadas no âmbito da Rede de Políticas de Saúde Pública brasileira e, de outro, os projetos de CSS e o PECS, nos faz compreender a centralidade da CSS para extensão da Rede de Saúde Pública para o ambiente internacional. Tal extensão teria o potencial de fortalecer nacionalmente e internacionalmente as posições portadas pela Rede de Políticas de Saúde. Nesse sentido, a manutenção da capacidade de implementação de projetos de CSS, particularmente do ponto de vista orçamentário, é variável crítica para a consolidação da extensão internacional da Rede.

3. A despeito da clara correlação entre projetos de cooperação técnica e o PECS/CPLP, pouco podemos afirmar acerca da efetividade da CSS brasileira em saúde e do próprio PECS, em razão da ausência de mecanismos de avaliação dos projetos. A análise resultante dessa pesquisa permite apenas identificar a percepção dos principais stakeholders em relação aos projetos em tela. Nos limites deste artigo, podemos afirmar que, enquanto projetos na área de construção de capacidades e treinamento são bem avaliados nos dois lados do Atlântico, projetos na área do complexo industrial de saúde e de vigilância encontram grandes dificuldades e, por vezes, resistências. As diferentes percepções identificadas sugerem a necessidade do tratamento analítico da articulação entre as Redes do Brasil e de Moçambique, de forma a identificar seus interesses e preferências particulares ${ }^{20}$.

${ }^{20}$ A esse respeito, ver Esteves e Assunção (2016, no prelo). 


\section{Quadro 2}

PECS/CPLP e Cooperação Técnica em Saúde, Brasil-Moçambique

\begin{tabular}{|c|c|}
\hline $\begin{array}{l}\text { PECS/CPLP: Eixos estratégicos e } \\
\text { redes estruturantes }\end{array}$ & $\begin{array}{l}\text { Projetos de cooperação em } \\
\text { saúde Brasil-Moçambique }\end{array}$ \\
\hline $\begin{array}{l}\text { EE1 - Treinamento e desenvolvimento de mão de } \\
\text { obra da área da saúde }\end{array}$ & $\mathrm{x}$ \\
\hline EE2 - Informação e comunicação na saúde & $\mathrm{x}$ \\
\hline EE3 - Pesquisa em saúde & $\mathrm{X}$ \\
\hline $\begin{array}{l}\text { EE4 - Desenvolvimento do complexo industrial } \\
\text { da saúde }\end{array}$ & $\mathrm{x}$ \\
\hline $\begin{array}{l}\text { EE5 - Vigilância epidemiológica e monitoramento } \\
\text { de situações da saúde }\end{array}$ & $\mathrm{X}$ \\
\hline \multicolumn{2}{|l|}{ EE6 - Emergências e desastres naturais } \\
\hline EE7 - Promoção e proteção da saúde & $\mathrm{x}$ \\
\hline RE1 - Institutos nacionais da saúde & $\mathrm{x}$ \\
\hline RE2 - Escolas nacionais de saúde pública & $\mathrm{X}$ \\
\hline RE3 - Escolas técnicas de saúde & $\mathrm{x}$ \\
\hline $\begin{array}{l}\text { RE4 - Centros técnicos de instalação e manutenção } \\
\text { de equipamentos }\end{array}$ & $\mathrm{x}$ \\
\hline
\end{tabular}

Fonte: Buss (2011). Elaboração própria.

\section{A cooperação em saúde com Angola ${ }^{21}$}

A cooperação Brasil-Angola no setor da saúde teve início em 1996, quando a ABC organizou uma missão com especialistas da saúde para discutir potenciais projetos em áreas definidas como estratégicas no âmbito da CPLP. Desde então, governos de países da África lusófona, incluindo Angola, vêm demandando apoio para seus sistemas nacionais de saúde. Durante os anos 1990, a ênfase da cooperação foi em treinamento e capacity building.

${ }^{21}$ Os resultados apresentados sinteticamente nesta seção encontram-se no relatório Brazilian health and agricultural cooperation in Angola: an overview, publicado em: <http:/ / bricspolicycenter.org/homolog/uploads/trabalhos/6838/doc/968418951.pdf>. 
De acordo com um documento fornecido pela Embaixada Brasileira em Luanda, em referência a julho de 2013, havia então somente dois projetos de cooperação bilateral em execução em Angola na área da saúde. Esses projetos eram também os únicos listados como "em execução" no site da ABC: (1) o Projeto Piloto em Doença Falciforme e (2) o Desenvolvimento de Capacidade para o Sistema de Saúde de Angola - Fase 2. Além desses, houve no período em tela a execução do projeto Proforsa, apresentado a seguir.

O Projeto Piloto em Doença Falciforme teve como objetivo reduzir a morbidez e a mortalidade relacionadas à essa doença em Angola, viabilizado por uma parceria entre o MS brasileiro e o Hospital Pediátrico David Bernardino. A doença falciforme é a doença hereditária mais comum no mundo, com altas taxas de morbidez e mortalidade. Mesmo que essa doença permaneça sem cura, muitas de suas manifestações e complicações são passíveis de 220 tratamento e prevenção, garantindo não só a sobrevivência, mas também melhor qualidade de vida para as pessoas por ela afetadas. Assim, o projeto visou à consolidação de políticas públicas direcionadas ao combate à doença e o emprego da triagem laboratorial neonatal e diagnóstico por imagem. O mesmo também buscou o desenvolvimento de capacidades técnicas relacionadas ao tratamento básico da doença e a restauração de lesões ósseas dela resultantes. O projeto teve um orçamento total de $291 \mathrm{mil}$ dólares, quase todo financiado pela $\mathrm{ABC}$ (17 mil dólares desse total foram advindos do MS brasileiro e 38 mil dólares do Hospital Pediátrico David Bernardino). O documento fornecido pela $\mathrm{ABC}$ relata atrasos e inconsistência na execução do projeto, fruto de problemas recorrentes de comunicação com as contrapartes angolanas.

Uma parceria entre a Fiocruz e o Ministério da Saúde de Angola resultou no projeto Desenvolvimento de Capacidade para o Sistema de Saúde de Angola - Fase 2. O projeto 
teve como objetivo fortalecer a capacidade de treinamento em saúde pública naquele país nos campos científico, tecnológico e educacional, incluindo comunicação e informação. As ações empreendidas pelo projeto foram direcionadas ao apoio do Ministério da Saúde de Angola no treinamento de profissionais para trabalhar no ensino, na pesquisa e na cooperação técnica na Escola de Saúde Pública de Angola. Além disso, o projeto buscou também estruturar uma rede de bibliotecas de saúde em Angola, reestruturar as escolas técnicas de saúde e fortalecer o Instituto Nacional de Saúde Pública. A primeira fase do projeto teve atividades incompletas/atrasadas enfrentando dificuldades na implementação relacionadas ao mestrado em Saúde Pública. Ainda assim, foi conferido grau de mestre em Saúde Pública a trinta profissionais angolanos e o programa de mestrado foi considerado pelo governo de Angola muito exitoso, manifestando interesse em apoiar a segunda edição do projeto ${ }^{22}$.

Apesar de não fazer parte da lista de projetos anteriormente mencionada, o Proforsa é certamente o projeto de maior visibilidade da cooperação brasileira em Angola na área da saúde pública. Como já dissemos, trata-se de uma iniciativa que envolve Brasil, Angola e Japão, e foi iniciada em 2011, com duração de três anos. O projeto teve como objetivo fortalecer as capacidades individuais, institucionais e sistêmicas do setor da saúde angolano. As instituições que lideraram o projeto foram a Escola Politécnica em Saúde

${ }^{22}$ É importante pontuar que apesar do status "em execução" atribuído aos projetos, a comunicação trocada com o MS brasileiro em setembro de 2014 a respeito do projeto da doença falciforme indicou várias inconsistências na sua execução, insinuando que ele foi finalizado sem que os parceiros tivessem completado as atividades anteriormente acordadas. O projeto da Fiocruz também parece ter sido finalizado, apesar de nenhuma parte angolana envolvida com o projeto em Luanda estivesse disponível para comentários. Ademais, não é improvável que, como em vários outros países, projetos de cooperação técnica sob negociação em Angola tenham sido paralisados devido aos severos cortes orçamentários tanto do Ministério das Relações Exteriores do Brasil quanto da Agência Brasileira de Cooperação. 
Joaquim Venâncio (EPSJV/Fiocruz) e Escola Nacional de Saúde Pública (ENSP/Fiocruz) para o componente de saúde primária, e Unicamp, através do Hospital Sumaré, e a JICA (Japan International Cooperation Agency) para o componente de saúde terciária ${ }^{23}$. No componente de saúde primária, o desenvolvimento de capacidades teve como foco quatro centros de referência em saúde em Luanda - Samba, Ingobota, Rangel e Ilha. Já no componente de saúde terciária, o projeto foi conduzido no Hospital Josina Machel e na Maternidade Lucrécia Paim.

Os desafios na execução do Proforsa, que transpareceram nas entrevistas, podem ser aglutinados em três categorias: (1) implementação, (2) financiamento e (3) contexto cultural e político. Um informante enfatizou como a Fiocruz tem sido hábil em promover o entendimento de questões básicas relacionadas ao sistema de saúde angolano entre os principais stakeholders do setor. Contudo, o mes222 mo informante criticou outras iniciativas top-down, como as implementadas por empresas privadas de consultoria em saúde, que impõem novas regras de gestão, diretivas e práticas sem discussão com os profissionais do setor. Entrevistas feitas com as contrapartes brasileiras relataram problemas financeiros. Cortes no orçamento do Ministério das Relações Exteriores afetaram de maneira significativa os projetos da $\mathrm{ABC}$ e forçaram a reestruturação de responsabilidades nas despesas do terceiro ano do Proforsa. Grande parte dos custos foi absorvida pela JICA, e uma parte menor, porém significativa, absorvida pelo Ministério da Saúde de Angola.

\footnotetext{
${ }^{23}$ Os serviços de saúde são classificados de acordo com o grau de complexidade tecnológica requerida aos procedimentos realizados. A atenção primária à saúde se refere à atenção básica, com ações de prevenção e proteção à saúde, com ações precoces que potencializem a intervenção ou mesmo evite sua necessidade. A atenção secundária se refere à atenção de média complexidade, realizada através de centros de atendimentos. A atenção terciária constitui alta complexidade, realizada através de serviços ambulatoriais e hospitalares especializados.
} 


\section{Quadro 3}

PECS/CPLP e Cooperação Técnica em Saúde, Brasil-Angola

\begin{tabular}{|c|c|}
\hline $\begin{array}{l}\text { PECS/CPLP: Eixos estratégicos e } \\
\text { redes estruturantes }\end{array}$ & $\begin{array}{l}\text { Projetos de cooperação em } \\
\text { saúde Brasil-Angola }\end{array}$ \\
\hline $\begin{array}{l}\text { EE1 - Treinamento e desenvolvimento de mão de } \\
\text { obra da área da saúde }\end{array}$ & $x$ \\
\hline \multicolumn{2}{|l|}{ EE2 - Informação e comunicação na saúde } \\
\hline \multicolumn{2}{|l|}{ EE3 - Pesquisa em saúde } \\
\hline \multicolumn{2}{|l|}{$\begin{array}{l}\text { EE4 - Desenvolvimento do complexo industrial da } \\
\text { saúde }\end{array}$} \\
\hline \multicolumn{2}{|l|}{$\begin{array}{l}\text { EE5 - Vigilância epidemiológica e monitoramento de } \\
\text { situações da saúde }\end{array}$} \\
\hline \multicolumn{2}{|l|}{ EE6 - Emergências e desastres naturais } \\
\hline EE7 - Promoção e proteção da saúde & $x$ \\
\hline \multicolumn{2}{|l|}{ RE1 - Institutos nacionais da saúde } \\
\hline RE2 - Escolas nacionais de saúde pública & $x$ \\
\hline RE3 - Escolas técnicas de saúde & $x$ \\
\hline $\begin{array}{l}\text { RE4 - Centros técnicos de instalação e manutenção } \\
\text { de equipamentos }\end{array}$ & \\
\hline
\end{tabular}

Fonte: Buss (2011). Elaboração própria.

Por fim, houve grande divergência em relação ao Caderno de Saúde Materno-Infantil. Abordagens distintas de brasileiros e japoneses terminaram por minar a confiança entre as partes e o recuo dos cooperantes brasileiros que decidiram não participar da elaboração do caderno. Paradoxalmente, tal caderno foi considerado por muitos stakeholders do setor da saúde, não diretamente envolvidos nesse projeto de cooperação triangular, como o principal resultado do Proforsa.

A análise da CSS brasileira no campo da saúde pública em Angola reflete um cenário bastante distinto daquele encontrado em Moçambique. Se contrastado com o 
PECS/CPLP para Moçambique, o portfólio de projetos em Angola é muito menos consistente (ver Quadro 3).

***

A partir das experiências nos âmbitos mini e multilateral, bem como em projetos de CSS, a Rede de Políticas de Saúde Pública cunhou o conceito de cooperação estruturante para caracterizar aquilo que seria a singularidade de suas práticas cooperativas. $\mathrm{O}$ conceito foi rapidamente apropriado por outras redes de políticas e passou a ser mobilizado também pela ABC. Contudo, a despeito de sua ampla difusão entre as redes de políticas da cooperação brasileira, a noção de cooperação estruturante ainda carece de precisão conceitual. Enquanto alguns agentes a relacionam a volume de recursos, outros identificam a cooperação estruturante como a construção de capacidades. Não obstante a 224 dispersão conceitual, pudemos observar que essa Rede oferece uma conceituação mais robusta. De fato, nessa chave, a cooperação estruturante se aproxima da ideia de construção de sistemas nacionais autônomos (self-reliance), capazes de formular e executar políticas em determinado setor, alinhadas às demandas específicas de seu respectivo contexto. No caso das Redes de Políticas de Saúde Pública, essa perspectiva informa ainda a construção de redes internacionais estruturantes em torno das diversas unidades que compõem ou deveriam compor os sistemas nacionais (collective self-reliance).

No caso da saúde, no âmbito da CPLP, essas redes incluem Institutos Nacionais de Saúde, Escolas Nacionais de Saúde Pública, Escolas Técnicas de Saúde e Bancos de Leite Humano, evidenciando extensões das redes e dos sistemas brasileiros. A Rede de Políticas de Saúde Pública representa, provavelmente, o exemplo mais bem desenvolvido da extensão internacional de caráter estruturante. 


\section{Quadro 4}

PECS/CPLP e CSS em Saúde em Angola e Moçambique

\begin{tabular}{|l|c|c|}
\hline \multicolumn{1}{|c|}{$\begin{array}{c}\text { PECS/CPLP: Eixos estratégicos } \\
\text { e redes estruturantes }\end{array}$} & $\begin{array}{c}\text { Projetos de } \\
\text { cooperação em } \\
\text { saúde Brasil-Angola }\end{array}$ & $\begin{array}{c}\text { Projetos de } \\
\text { cooperação em saúde } \\
\text { Brasil-Moçambique }\end{array}$ \\
\hline $\begin{array}{l}\text { EE1 - Treinamento e desenvolvimento } \\
\text { de mão de obra da área da saúde }\end{array}$ & $\mathrm{X}$ \\
\hline $\begin{array}{l}\text { EE2 - Informação e comunicação na } \\
\text { saúde }\end{array}$ & & $\mathrm{X}$ \\
\hline EE3 - Pesquisa em saúde & $\mathrm{X}$ \\
\hline $\begin{array}{l}\text { EE4 - Desenvolvimento do complexo } \\
\text { industrial da saúde }\end{array}$ & $\mathrm{X}$ \\
\hline $\begin{array}{l}\text { EE5 - Vigilância epidemiológica e } \\
\text { monitoramento de situações da saúde }\end{array}$ & & $\mathrm{X}$ \\
\hline EE6 - Emergências e desastres naturais & $\mathrm{X}$ & $\mathrm{X}$ \\
\hline EE7 - Promoção e proteção da saúde & & $\mathrm{X}$ \\
\hline RE1 - Institutos nacionais da saúde & $\mathrm{X}$ & $\mathrm{X}$ \\
\hline RE2 - Escolas nacionais de saúde pública & $\mathrm{X}$ & $\mathrm{X}$ \\
\hline RE3 - Escolas técnicas de saúde & & \\
\hline $\begin{array}{l}\text { RE4 - Centros técnicos de instalação e } \\
\text { manutenção de equipamentos }\end{array}$ & & \\
\hline
\end{tabular}

Fonte: Buss (2011). Elaboração própria.

O Quadro 4 permite comparar dimensões da extensão internacional das redes e os projetos de cooperação Sul-Sul em saúde em Angola e Moçambique.

Esse quadro nos proporciona revisitar as conclusões preliminares apresentadas anteriormente e avançá-las em termos de conclusões finais. Como afirmamos, os processos de elaboração de declarações e planos nos âmbitos mini e multilateral, de um lado, e os projetos de CSS, de outro, parecem se retroalimentar. Os casos estudados nos permitiram identificar a tentativa de extensão internacional da Rede de Políticas de Saúde Pública brasileira a partir de 
planos estratégicos minilaterais e de projetos de CSS. Tal extensão carrega consigo, como procuramos argumentar, escolhas constitucionais, como acesso universal, e escolhas de políticas, que vinculam saúde pública e desenvolvimento de forma abrangente. Tal extensão reforçaria tanto doméstica como internacionalmente as posições da Rede brasileira em áreas diversas como a de acesso a medicamentos.

Contudo, a análise nos fez perceber que, a despeito dos esforços empreendidos pelos cooperantes brasileiros em saúde, suas ações se encontram severamente constrangidas, tanto por limites orçamentários como pela ausência de um quadro legal que lhes permita operar. Tais constrangimentos têm severas consequências em termos de efetividade e confiança e sobre a capacidade de manutenção das extensões internacionais da Rede, como pudemos compreender a partir das entrevistas realizadas.

Finalmente, a diferença significativa entre os casos de 226 Angola e Moçambique, bem como as diferentes percepções acerca dos projetos de CSS em ambos os casos, apontam para a necessidade de aprofundamento das pesquisas de campo em torno da questão específica da articulação entre cooperantes brasileiros e de países terceiros. Tal análise é necessária para que possamos identificar as variáveis que incidem sobre o processo de extensão internacional da Rede e compreender a dinâmica da cooperação estruturante brasileira.

\section{Paulo Esteves}

é professor do Instituto de Relações Internacionais da Pontifícia Universidade Católica do Rio de Janeiro (PUC-Rio) e pesquisador do BRICS Policy Center, do CNPq e da Faperj (CNE), e do projeto Brazil's Rise to the Global Stage (BraGS): Humanitarism, Peacekeeping and Quest for Great Powerhood (PRIO). 


\section{João Fonseca}

é mestre em Relações Internacionais pela PUC-Rio, consultor do Banco Mundial em Moçambique e pesquisador associado do BRICS Policy Center.

\section{Geovana Zoccal Gomes}

é doutoranda em Relações Internacionais pela PUC-Rio e pesquisadora do BRICS Policy Center.

\section{Bibliografia}

ALMEIDA, C. 2006. Health sector reform in Latin America and the Caribbean: the role of international organizations in formulating agendas and implementing policies. Well-being and Social Policy, n. 2, pp. 123-60.

ALMEIDA, C. M. et al. 2010. A concepção brasileira de "cooperação SulSul estruturante em saúde”. Revista Eletrônica de Comunicação, Informação e Inovação em Saúde, v. 4, n. 1, pp. 25-35.

ANVISA - Agência Nacional de Vigilância Sanitária. 2008. Banco de Leite Humano: Funcionamento, Prevenção e Controle de Riscos. Brasília: Anvisa. BÖRZEL, T. J., 1998. Organizing Babylon: on the different conceptions of policy networks. Public Administration, v. 76, n. 2, pp. 253-73.

BRASIL. Ministério das Relações Exteriores. Divisão de Temas Educacionais. 2013. Divisão de Temas Educacionais. Disponível em: $<$ http://www.dce.mre.gov.br/index.php>.

BRASIL. Ministério da Saúde. 1986. Relatório Final da $8^{a}$ Conferência Nacional de Saúde. Brasília: Centro de Documentação do Ministério da Saúde. BRENNAN, G.; BUCHANAN, J. M. 1985. The reason of rules: constitutional political economy. Cambridge/New York: Cambridge University Press. BRUYN, T. D. 2014. Brazil, India, China and South Africa in the Health Sector in Mozambique. Challenging the status quo? The impact of the emerging economies on the global governance of development cooperation, 4th paper, KU Leuven and HIVA.

BUCHANAN, J. M. 1987. Constitutional economics. In: EATWELL, J.; MILLGATE, M.; NEWMAN, P. The new Palgrave dictionary of economics. London: Macmillan. 1990. The domain of constitutional economics. Constitutional Political Economy, v. 1, n. 1, pp. 1-18.

BUCHANAN, J. M.; TULLOCK, G. 1962. The calculus of consent. Ann Arbor: University of Michigan Press. 
BUSS, P. 2011. Brasil: estruturando a cooperação na saúde. The Lancet, Saúde no Brasil, v. 311, n. 9779, pp. 1722-723.

BUSS, P. M.; FERREIRA, J. R. 2010. Ensaio crítico sobre a cooperação internacional em saúde. RECIIS - Revista eletrônica de comunicação, informação \& inovação em saúde, v. 4, n. 1, pp. 93-105.

BUSS, P. M.; PELLEGRINI FILHO, A. 2007. A saúde e seus determinantes sociais. Physis, v. 17, n. 1, pp. 77-93.

CABRAL, L.; WEINSTOCK, J. 2010. Brazilian technical cooperation for development: drivers, mechanics and future prospects. London: ODI.

COOPER, A. F.; FAROOQ, A. B. 2015. Testando a cultura de clube dos brics: a evolução de um novo banco de desenvolvimento. Contexto Internacional, v. 37, n. 1, pp. 13-46.

CPLP - Comunidade dos Países de Língua Portuguesa. 2009. Plano Estratégico de Cooperação em Saúde da CPLP (PECS/CPLP) 2009-2012. Estoril, Portugal: CPLP.

ESCOREL, S. 1999. Reviravolta na saúde: origem e articulação do movimento sanitário. Rio de Janeiro: Fiocruz.

ESCOREL, S.; NASCIMENTO, D. R.; EDLER, F. C. 2005. As origens da Reforma Sanitária e do SUS. In: LIMA, N. T. et al. Saúde e democracia: história e perspectivas do SUS. Rio de Janeiro: Fiocruz.

228 ESTEVES, P.; ASSUNÇÃO, M. 2016 [no prelo]. The partnership puzzle: Brazilian health development practices in Mozambique. In: BERGAMASCHI, I.; MOORE, P.; TICKNER A. B. South-South cooperation beyond the myth (forthcoming).

FARIA, C. A. 2012. A difusão de políticas sociais como estratégia de inserção internacional: Brasil e Venezuela comparados. Interseções, v. 14, n. 2, pp. 335-71.

FIOCRUZ - Fundação Oswaldo Cruz. 2012. A saúde no Brasil em 2030 prospecção estratégica do sistema de saúde brasileiro: desenvolvimento, Estado e políticas de saúde. Rio de Janeiro: Fiocruz/Ipea/Ministério da Saúde/ Secretaria de Assuntos Estratégicos da Presidência da República.

GADELHA, C. A. G. 2006. Development, health-industrial complex and industrial policy. Revista de Saúde Pública, v. 40, número especial, São Paulo. HOCHMAN, G. 1988. Aprendizado e difusão na constituição de políticas: a previdência social e seus técnicos. RBCS - Revista Brasileira de Ciências Sociais, n. 7, pp. 84-97. 2007. Agenda internacional e políticas nacionais: uma comparação histórica entre programas de erradicação da malária e da varíola no Brasil. In: HOCHMAN, G.; ARRETCHE, M.; MARQUES, E. Políticas públicas no Brasil. Rio de Janeiro: Fiocruz. 
IPEA/ABC - Instituto de Pesquisa Econômica Aplicada/Agência Brasileira de Cooperação. 2010. Cooperação Brasileira para o Desenvolvimento Internacional: 2005-2009. Brasília: Ipea.

KICKBUCH, I.; SILBERSCHMIDT, G.; BUSS, P. M. 2007. Global health diplomacy: The need for new perspectives, strategic approaches and skills in global health. Bull WHO, v. 85, n. 3, pp. 230-32.

LANA, X.; EVANS, M. 2004. Policy transfer between developing countries: the transfer of the Bolsa-Familia programme to Ecuador. In: EVANS, M. Policy transfer in global perspective. Burlington: Ashgate.

LEITE, I. C.; SUYAMA, B.; WAISBICH, L. T. 2013. Policy Brief. Para além do tecnicismo: a cooperação brasileira para o desenvolvimento internacional e caminhos para a sua efetividade e democratização. In: CEBRAP/ IDS - INSTITUT OF DEVELOPMENT STUDIES/ARTICULAÇÃO SUL. Disponível em: <http://www.cebrap.org.br/v2/files/upload/ biblioteca_virtual/item_796/26_08_13_14Policy_Briefing_Para\%20 al\%C3\%A9m\%20do\%20tecnicismo.pdf>. Acesso em: 24 jul. 2016.

LIMA, N. T.; FONSECA, C.; HOCHMAN, G. 2005. Saúde na construção do Estado Nacional no Brasil: reforma sanitária em perspectiva histórica. In: LIMA, N. T. et al. Saúde, democracia, história e perspectivas do SUS. Rio de Janeiro: Fiocruz.

MARSH, D.; RHODES, R. A. 1992. Policy networks in British government. Oxford: Oxford University Press.

MATTOS, R. 2001. As agências internacionais e as políticas de saúde nos anos 90: um panorama geral da oferta de ideias. Ciência Ẽ Saúde Coletiva, v. 6, n. 2, pp. 377-89.

MELO, M. A. 2004. Escolha institucional e a difusão dos paradigmas de política: o Brasil e a segunda onda de reformas previdenciárias. Dados, v. 47, n. 1, pp. 169-206.

MILANI, C.; LOPES, R. N. 2014. Cooperação Sul-Sul e policy transfer em saúde pública: análise das relações entre Brasil e Moçambique entre 2003 e 2012. Carta Internacional, v. 9, n. 1, pp. 59-78.

NAIM, M. 2009. Minilateralism: The magic number to get real international action. Foreign Policy, n. 173, pp. 135-36.

O BRASIL e a Cooperação Sul-Sul. 2013. Colóquio realizado em 28 jun. BRICS Policy Center/MTE/ABC - Ministério das Relações Exteriores/ Agência Brasileira de Cooperação, Rio de Janeiro. Disponível em: $<$ http:/ /bricspolicycenter.org/homolog/arquivos/e.pdf > . Acesso em: 24 jul. 2016.

RHODES, R. A. 1988. Beyond Westminster and Whitehall: the sub-central governments of Britain. Unwin Hyman, Great Britain. 
2006. Policy network analysis. In: REIN, M.; GOODIN, R. E.; MORAN, M. The Oxford handbook of public policy. Oxford: Oxford University Press.

RUSSO, G.; CABRAL, L. V.; FERRINHO, P. 2013. Brazil-Africa technical cooperation in health: what's its relevance to the post-Busan debate on aid effectiveness. Globalization and Health, v. 9, n. 2.

SUGYIAMA, N. B. 2011. Bottom-up policy diffusion: national emulation of a conditional cash transfer program in Brazil. Publius: The Journal of Federalism, v. 1, n. 27, pp. 1-27.

TEMPORÃO, J. G. 2009. Pronunciamento do Ministro da Saúde do Brasil, Dr. José Gomes Temporão, na reunião constitutiva do Plano Estratégico de Cooperação em Saúde da Comunidade de Países de Língua Portuguesa (CPLP).

VISSER-VALFREY, M.; UMARJI, M. B. 2010. Sector budget support in practice case study: health sector in Mozambique. Overseas Development Institute. London.

WORLD BANK. 1987. Financing health services in developing countries: an agenda for reform. Washington, DC: World Bank. 


\section{A REDE DE POLÍTICAS DE SAÚDE PÚBLICA E A COOPERAÇÃO SUL-SUL: OS CASOS DE MOÇAMBIQUE E ANGOLA}

\section{PAULO ESTEVES}

GEOVANA ZOCCAL GOMES

JOÃO MOURA FONSECA

Resumo: Análises da cooperação brasileira para o desenvolvimento frequentemente tomam o Ministério das Relações Exteriores e a Agência Brasileira de Cooperação como ponto de partida institucional. Contudo, pesquisas de campo realizadas pelos autores no Brasil, Angola e Moçambique sugerem ser necessário conferir maior ênfase analítica ao papel das chamadas agências executoras e das redes de políticas de que fazem parte. Com foco no setor da saúde, este artigo parte da Rede de Políticas de Saúde Pública para então analisar as ações de cooperação brasileira em Moçambique e Angola. Baseado nos processos de constituição e consolidação dessa rede, o argumento avança no sentido de que o engajamento dos agentes da rede em projetos de cooperação Sul-Sul deve ser compreendido de forma mais ampla, paralela à da extensão internacional da própria rede, elemento que pode ser verificado no portfólio de projetos brasileiros na área da saúde nos dois países africanos. Conclusões alcançadas com essa abordagem sinalizam, do ponto de vista da formulação de políticas, a necessidade de consolidação do que os cooperantes brasileiros do campo da saúde denominam cooperação estruturante. $\mathrm{O}$ texto defende, ainda, maior investimento em trabalhos de campo como meio para melhor compreender a articulação entre cooperantes brasileiros e seus parceiros na África lusófona.

Palavras-chave: Cooperação Sul-Sul; Saúde; Rede de Políticas Públicas; Moçambique, Angola. 


\section{THE PUBLIC HEALTH POLICY NETWORK AND SOUTH-SOUTH COOPERATION: THE CASES OF MOZAMBIQUE AND ANGOLA}

Abstract: Analyses of Brazilian development cooperation often take the Ministry of Foreign Affairs and the Brazilian Cooperation Agency as an institutional starting point. However, field research conducted by the authors in Brazil, Angola, and Mozambique suggest that it is necessary to provide greater analytical emphasis to the role played by executing agencies and policy networks of which executing agencies are part. Focusing on the health sector, this article takes the Public Health Policy Network as the basis for analyzing Brazilian cooperation in health in Mozambique and Angola. By analyzing the processes of formation and consolidation of the Public Health Policy Network, it is argued that the involvement of its agents in South-South cooperation projects must be understood in light of a broader process of international extension of their own policy network, an element that can be found in the portfolio of Brazilian health projects in the two African countries. Conclusions reached through this approach point, from the perspective of policy-making, to the need for consolidating what Brazilian copers term structuring cooperation. Increased investment in field work is required to expand our understanding of relationships between Brazilian copers and their partners in Lusophone Africa.

Keywords: South-South Cooperation; Health; Public Policies Network; Mozambique; Angola.

Recebido em: 12/4/2016 Aprovado em: 17/6/2016 